\title{
Search for critical points in the SU(2) Higgs model
}

\author{
W. Bock \\ Institut für Theoretische Physik, Rheinish-Westfälische Technische Hochschule Aachen, \\ D-5100 Aachen, Federal Republic of Germany \\ and HLRZ c/o Forschungszentrum Jülich, P.O. Box 1913, D-5170 Jülich, Federal Republic of Germany \\ H. G. Evertz \\ II. Physikalisches Institut, Universität Hamburg, D-2000 Hamburg, Federal Republic of Germany \\ J. Jersák \\ Institut für Theoretische Physik, Rheinish-Westfälische Technische Hochschule Aachen, \\ D-5100 Aachen, Federal Republic of Germany \\ and HLRZ c/o Forschungszentrum Jülich, P.O. Box 1913, D-5170 Jülich, Federal Republic of Germany \\ D. P. Landau \\ Center for Simulational Physics, University of Georgia, Athens, Georgia 30602 \\ T. Neuhaus \\ Institut für Theoretische Physik, Universität Bielefeld, D-4800 Bielefeld, Federal Republic of Germany \\ J. L. Xu \\ Center for Simulational Physics, University of Georgia, Athens, Georgia 30602
}

(Received 24 July 1989)

\begin{abstract}
We study the order of the Higgs phase transition in the SU(2) Higgs model at several values of the gauge coupling $\beta$ for bare quartic coupling $\lambda=0.5$ using Monte Carlo simulations. We determine the internal energy of metastable states on various lattice sizes and estimate that the transition terminates at the critical point located at $1.95<\beta_{c}<2.25$. We also apply a recently proposed multihistogram method in our data analysis. At $\beta=2.25$ and $\beta=2.4$ our data strongly indicate the presence of a first-order phase transition. For $\beta \geq 2.6$ we find no indication of a discontinuity in the internal energy on lattices up to size $16^{4}$ and the transition is either weakly first order or of higher order.
\end{abstract}

\section{INTRODUCTION}

The Glashow-Weinberg-Salam (GWS) gauge theory of electroweak interactions assumes the presence of the Higgs mechanism for its successful description of many phenomena. The latter is responsible for the generation of vector-boson masses, due to the coupling of the gauge fields to the self-interacting scalar fields. The Higgs sector of the theory (coupled gauge and scalar fields without fermions) is, however, not yet completely understood. One important open question is-Does a nontrivial field theory exist in the infinite-cutoff limit? Also, the nonperturbative effects of a phenomenologically allowed strong self-coupling of the scalar field should be studied. Therefore various Higgs models have been investigated in recent years by means of nonperturbative Monte Carlo simulations on the lattice. For a review and a list of references on lattice Higgs models see Ref. 1. This approach requires understanding the continuum limit of the lattice Higgs models; i.e., one has to find a suitable critical point. At such a point the system flows to a fixed point in the sense of the renormalization group and the limit of vanishing lattice constant can be performed.
One possible scenario is that the continuum limit relevant for the GWS theory is approached in the vicinity of the Gaussian fixed point of the scalar theory. It has been demonstrated then that the inclusion of the gauge fields cannot change the noninteracting character of the resulting continuum theory. ${ }^{2}$ An interacting Higgs sector is possible only if one introduces a finite cutoff, and the GWS theory is then an effective field theory valid only at energies lower than the intrinsic scale parameter. This approach has recently led to the determination of an upper bound for the Higgs-boson mass. ${ }^{3}$

Although this scenario is at present acceptable both from the theoretical and phenomenological points of view, it is also important to examine the Higgs models for nontrivial fixed points where a genuine continuum limit with interacting fields could be possible. It is now nearly certain that the pure scalar field theory in four dimensions does not have such a nontrivial fixed point, but we cannot exclude the possibility that the coupled system of gauge and scalar fields behaves differently. Higgs models have large manifolds of phase transitions in the space of couplings and the fixed-point structure has not yet been reliably determined. 
Motivated by these considerations we have investigated the nature of the phase transition in the SU(2) Higgs model with scalar fields in the fundamental representation. This model corresponds to the Higgs sector of the GWS theory if the $U(1)$ gauge coupling is neglected. The model is known to have a two-dimensional sheet of "Higgs" phase-transition (PT) points in the threedimensional space of coupling parameters $\beta, \kappa$, and $\lambda$ (here $\beta=4 / g^{2}$ is related to the gauge field coupling $g, \lambda$ is the bare quartic self-coupling, and $\kappa$ is the hopping parameter of the scalar field). For small $\lambda \leq 0.03$ and for $\beta=1.0-3.0$ the Higgs PT is distinctly of first order ${ }^{4}$ as suggested by perturbative calculations. ${ }^{5}$ When $\lambda$ or $\beta$ increase, the Higgs PT weakens and for the pure scalar field theory at $\beta=\infty$ the Higgs PT is of second order. It is possible, however, that the order of the Higgs PT at a fixed value of $\lambda$ changes at some finite $\beta$ thus implying the existence of a tricritical line separating a twodimensional manifold of critical points from the phase transitions of first order. For theoretical hints in this direction see Ref. 6 . In the vicinity of a possible tricritical line one could then hope to construct a nontrivial continuum limit.

From earlier numerical data $^{4}$ we concluded that $\lambda=0.5$ is a good candidate for a search for such an effect. At this $\lambda$ the first order of the Higgs PT is still observable for $\beta=2.25$ but the Higgs PT is weak enough that one can expect a possible change of the order with increasing but still moderate values of $\beta$. Therefore we have fixed $\lambda$ to this value throughout the paper.

In addition, for large values of $\lambda$ the Higgs PT sheet is bounded by a line of critical points at small positive values of $\beta$. Thus a nontrivial continuum limit might also be possible there. Although its relevance for the GWS theory is not obvious, such a continuum limit might be of fundamental field-theoretical interest. The localization of these end points is also interesting from the point of view of recently performed investigations of the $\mathrm{SU}(2)$ Higgs model coupled to fermions. ${ }^{7}$ There it is desirable to compare the positions of the Higgs and the chiral phase transitions in order to find out whether they coincide.

The scenarios for continuum limits just described might be unlikely. However, their possible impact on the GWS theory nevertheless justifies their careful examination. We face very difficult problems when using the standard Monte Carlo simulation for this purpose because it is very difficult to distinguish weakly first-order phase transitions from second-order ones on a finite lattice. Previous studies have encountered such problems in the localization of critical points and/or tricritical points in spin systems ${ }^{8}$ and lattice gauge theories. ${ }^{9}$ At present we hope to gain better knowledge of the various properties of the Higgs PT and to localize regions in the coupling space where further investigations, e.g., using the Monte Carlo renormalization-group (MCRG) technique would be most promising.

\section{BACKGROUND}

\section{A. The model}

The lattice regularized action for the SU(2) Higgs model is given by the expression

$$
\begin{aligned}
S= & -\frac{\beta}{4} \sum_{P} \operatorname{tr}\left(U_{P}+U_{P}^{\dagger}\right) \\
& -\kappa \sum_{x} \sum_{\mu=1}^{4} \operatorname{Re}\left(\operatorname{tr} \Phi_{x}^{\dagger} U_{x, \mu} \Phi_{x+\mu}\right) \\
& +\lambda \sum_{x} \frac{1}{2} \operatorname{tr}\left(\Phi_{x}^{\dagger} \Phi_{x}-1\right)^{2}+\sum_{x} \operatorname{tr} \Phi_{x}^{\dagger} \Phi_{x}
\end{aligned}
$$

Here $U_{x, \mu}$ are link variables of the gauge field in the fundamental representation of the $\mathrm{SU}(2)$ gauge group and $U_{P}$ are their products along the lattice plaquettes. The site variables of the scalar field $\Phi_{x}$ are written in the form $\Phi_{x}=\rho_{x} \sigma_{x}$ where $\sigma_{x} \in \mathrm{SU}(2)$ is in the fundamental representation, too, and $\rho_{x}$ is the length of the scalar field. The first term describes the pure gauge field interaction; the second one the interaction between the scalar and the gauge fields and the last one the self-interaction of the scalar fields. The model has the coupling constants $\beta=4 / g^{2}$ with $g$ being the gauge coupling constant, $\kappa$ the hopping parameter, and $\lambda$ the bare quartic self-coupling, which is fixed to the value 0.5 throughout this paper. The system has only one phase: the confinement-Higgs phase. There is a Higgs PT line separating the confinement region below the phase transition (smaller values of $\kappa$ ) from the region above, which is the Higgs region. The line extends from $\beta=\infty$ [where the model reduces to the pure $O(4)$-symmetric $\Phi^{4}$ theory] to small values of $\beta$, but has a critical end point at some finite $\beta$ (Refs. 4 and 10).

\section{B. Details of simulation}

The simulations were carried out on Cyber 205 vector computers using the Metropolis algorithm on $L^{4}$ hypercubic lattices with periodic boundary conditions. While most of our runs were performed with a one-link update, we also performed runs with three hits for larger values of $\beta(\beta \geq 2.6)$ thus achieving an increased acceptance rate. In order to minimize the working space requirements we simulated the icosahedral finite subgroup of $\mathrm{SU}(2)$ which is a very good approximation to $\mathrm{SU}(2)$ for the values of the couplings we have considered. ${ }^{4} \mathrm{~A}$ fully vectorized code for the updating using a checkerboard decomposition of $L^{4}$ lattices, was implemented. We used two different random-number generators: one based on the congruent modulus method ${ }^{11}$ and second a shift register random-number generator. No systematic difference in our results was observed. Typically we discarded $10^{4}$ sweeps at the beginning, allowing for equilibration. Relatively far (in the $\kappa$ direction) from the phase transition $10^{4}$ sweeps were kept for measurements; close to the Higgs PT we performed up to $10^{5}$ sweeps even on our largest lattice. We considered lattices ranging in size from $4^{4}$ to $16^{4}$ and performed our simulation at many values of the couplings using a total of about $10^{3} \mathrm{~h}$ supercomputer time.

\section{Finite-size effects}

Phase transitions are extensively studied in the framework of statistical mechanics. The properties of the internal energy determine the nature of the phase transi- 
tions. The internal energy develops a gap at a first-order phase transition in the infinite-volume limit, while at a second-order phase transition the internal energy is continuous, but its derivative with respect to its couplings becomes infinite. Performing numerical simulations on a finite lattice poses the problem of extrapolating finitevolume data in order to determine infinite-volume properties of the internal energy.

In our model we concentrated on the hopping term, which is the interaction term of the scalar and gauge fields

$$
E=\frac{1}{8} \frac{1}{L^{4}} \sum_{x} \sum_{\mu=1}^{4} \operatorname{Re}\left(\operatorname{tr} \Phi_{x}^{\dagger} U_{x, \mu} \Phi_{x+\mu}\right) .
$$

Its expectation value $\langle E\rangle$ is the part of the internal energy contained in the hopping term of the action. This choice is motivated by our earlier ${ }^{4}$ observation, that the operator (2) most clearly exhibits the critical behavior at the Higgs PT.

Various methods can be devised in order to study phase transitions in different situations. At a strong firstorder phase transition one can study the appearance of long-living metastable states in the computer time sequence of generated $E$ values $E_{i}$, which manifest themselves as double-peak structures in the distribution function $N(E)$ of $E_{i}$. It is straightforward to estimate the gap in the internal energy

$$
\Delta E=E_{+}-E_{-}
$$

by locating the maxima $E_{+}$and $E_{-}$in the distribution function $N(E)$ on each lattice and at values of couplings where the two metastable states are equally probable. For a strong first-order phase transition one finds a gap which is insensitive to a variation of the lattice size, making the determination of the first-order nature of the phase transition with numerical methods relatively easy. We emphasize that on a finite lattice the internal energy $\langle E\rangle$ is a continuous function of the coupling constants although metastable states are present in the system. This becomes apparent numerically when the statistics of the simulation is high enough (rounding effect). It is only in the infinite-volume limit, when the Monte Carlo lifetime of the metastable states becomes infinite, that the internal energy develops a discontinuity.

The situation, however, becomes much more complicated if the transition is either of weak first order or of second order, but close to a first-order line. Here the locations of possible double peaks $E_{+}$and $E_{-}$and the gap size $\Delta E$ become functions of the lattice size and even a large gap on a small lattice can decrease to a small value if one increases the lattice size. It is then naturally very hard to decide from a numerical simulation what its value is in the infinite-volume limit. It may even happen that a double-peak distribution function $N(E)$ on a finite lattice approaches a Gaussian form in the thermodynamic limit and the phase transition is actually of second order. Such phenomena can be observed in the vicinity of tricritical points and critical points.

In our simulation we monitored the computer time evolution of values $E_{i}$ calculated in single configurations separated by 10 sweeps. Typically the statistics at the phase transition points was large enough to allow a few phase flips or fluctuations of the system, even on larger lattices. We then determined the distribution function $N(E)$ by binning the obtained values of $E_{i}$ into 50 bins and determined $E_{+}$and $E_{-}$from the location of the maxima, if they were present.

We also tried to refine this approach by replacing the sequence of values $E_{i}$ by a sequence of smoothed values $E_{j}^{*}$. We calculated the averages of $E_{i}$ values in blocks with length $N_{S}$ and with the center at $j$,

$$
E_{j}^{*}=\frac{1}{N_{S}+1} \sum_{|j-i| \leq N_{S} / 2} E_{i},
$$

and determined the corresponding distribution functions $N^{*}\left(E^{*}\right)$. This approach is motivated by the idea that one should be able to eliminate fluctuations on short scales in computer time but still preserve the dynamics of the phase flips. This is especially useful if the fluctuations in each metastable state are not much smaller than the gap size itself. The distribution function $N^{*}\left(E^{*}\right)$ of the smoothed $E$ values can then reveal the locations $E_{+}$and

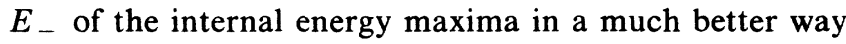
than $N(E)$.

The second moment of the distribution function-the specific heat-measures the fluctuations of the internal energy around its average value

$$
C=\partial_{\kappa}\langle E\rangle=8 L^{4}\left\langle(E-\langle E\rangle)^{2}\right\rangle \text {. }
$$

Its finite-size scaling analysis has been the standard tool for the study of second-order phase transitions. ${ }^{12}$ In particular one can determine $C$ as a function of $\kappa(\kappa$ plays the role of the inverse temperature for fixed values of $\lambda$ and $\beta$ ) and then study its maximal values $C_{\max }$ as a function of the lattice size $L$. The divergence of the specific heat with $L$ is described by the scaling law

$$
C_{\max } \propto L^{\alpha / v}
$$

for very large lattices with critical exponents $\alpha$ and $v$. We note that $C_{\max }$ diverges even at a first-order phase transition: the fluctuations between the metastable states dominate the fluctuations of the internal energy and the specific heat diverges with the volume of the system, ${ }^{13}$ i.e., $C_{\max } \propto L^{4}$ in our case. We determined the specific heat by means of the second expression in Eq. (5) and its error was determined by blocking the data for $C$ into few blocks of typical length 1000-5000 sweeps. Those entries were then treated as independent measurements.

We also considered the modified fourth cumulant $V_{L}$ defined by

$$
V_{L}=1-\frac{\left\langle E^{4}\right\rangle_{L}}{3\left\langle E^{2}\right\rangle_{L}^{2}}
$$

This quantity behaves quite differently at first- and second-order phase transitions. At a second-order phase transition its value in the thermodynamic limit is $\frac{2}{3}$ (reflecting the fact that the corresponding distribution functions approach Gaussian forms), this value is more- 
over independent of the temperature. For a first-order phase transition, however, $V_{L}$ will have a value smaller than $\frac{2}{3}$ on the phase transition point in the thermodynamic limit. This property and the finite-size dependence of $V_{L}$ has been successfully used in statistical mechanics models to study the order of phase transitions. ${ }^{13,14}$

\section{Multihistogram method}

It is very difficult in numerical simulations to reliably determine, e.g., the maximum of the specific heat $C_{\max }$ on a given lattice because the precise location of the PT is usually not known. While one can obtain a reasonable estimate by performing a large number of MC runs in the close vicinity of the PT (in our model we chose a set of $\kappa_{\gamma}$ with $\gamma=1, \ldots, N$ at fixed $\beta$ ), the problem of principle remains: it is most likely that the MC simulation misses the finite-volume phase-transition point.

Recently Ferrenberg and Swendsen (FS) proposed a method which allows an analytic continuation of thermodynamic quantities, e.g., the specific heat, in the critical region of the model. ${ }^{15}$ Making use of the analytic form of the partition function, and combining the measured distribution functions $N_{\gamma}(E)$ as determined in the MC simulation at couplings $\kappa_{\gamma}$, the probability distribution function $P(E, \kappa)$ is determined by means of the equation

$$
P(E, \kappa)=\frac{\sum_{\gamma=1}^{N} N_{\gamma}(E) \exp \left(8 \kappa L^{4} E\right)}{\sum_{\gamma=1}^{N} n_{\gamma} \exp \left(8 \kappa_{\gamma} L^{4} E-F_{\gamma}\right)} .
$$

Here is $n_{\gamma}$ the total number of entries into the (unnormalized) histogram at $\kappa_{\gamma}$ and independent MC measurements are assumed. The set of free energies $F_{\gamma}$ with $\gamma=1, \ldots, N$ can be determined self-consistently by Eq. (8) and

$$
\exp \left(F_{\gamma}\right)=\sum_{E} P\left(E, \kappa_{\gamma}\right) .
$$

The specific heat can then be calculated by means of

$$
C(\kappa)=8 L^{4} \sum_{E} P(E, \kappa)\left(E^{2}-\langle E\rangle^{2}\right) .
$$

Analogous formulas hold for the mean of the internal energy $\langle E\rangle$ and the modified fourth cumulant $V_{L}$. An estimate of the errors can be obtained by applying the multihistogram method for different subsets of $\kappa_{\gamma}$ values.

\section{RESULTS AND DISCUSSION}

We studied the model (1) for $\lambda=0.5$ at values $\beta=1.95$, $2.1,2.25,2.4,2.6$, and 3.5 varying the hopping parameter $\kappa$. Figure 1 shows $\langle E\rangle$ on the $16^{4}$ lattice in the vicinity of the Higgs PT. In the cases of $\beta=2.1$ and 2.25 we observe a rapid variation of $\langle E\rangle$ as we cross the phase transition whereas for the larger $\beta$ values and also for $\beta=1.95$ the curves are much smoother. We expect that at $\beta=2.4,2.6$, and 3.5 , in spite of this smoothness, the Higgs PT is present, as this transition is believed to continue to $\beta=\infty$. At $\beta=1.95$, however, the data indicate
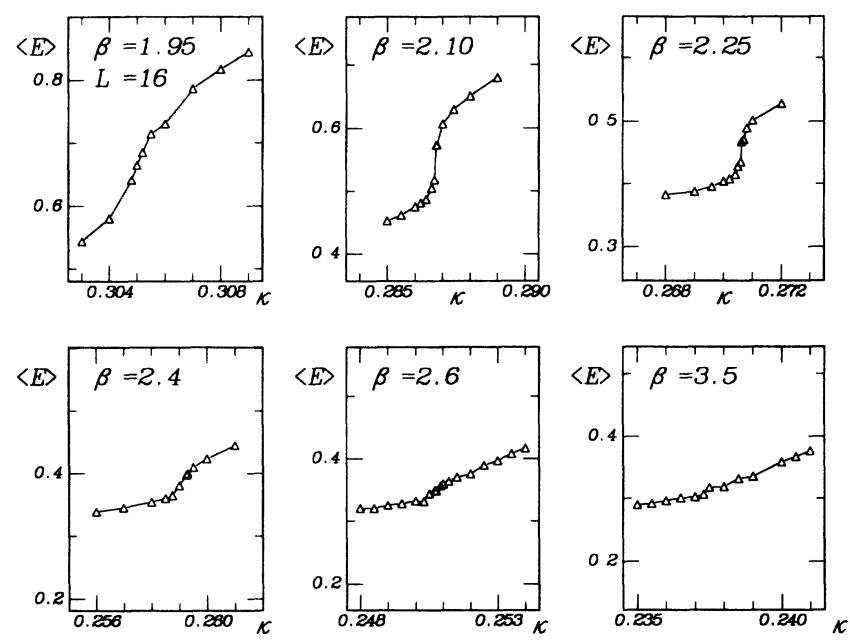

FIG. 1. The internal energy $\langle E\rangle$ as a function of $\kappa$ on $16^{4}$ lattices for various values of $\beta$.

the absence of a phase transition; one can only see a rounded, nondivergent specific-heat peak at $\kappa=0.305(1)$.

We determined the positions of the Higgs PT on the $16^{4}$ lattice from the distribution functions $N(E)$. At $\beta=2.1,2.25$, and 2.4 double peaks are present and it is possible to determine the metastability region. Its width allows us to estimate the error of the position of the Higgs PT points. At $\beta=2.6$ and 3.5 we did not observe metastability on our lattices and we therefore determined the location of the phase transition from the location of the peak of the specific heat. The phase-transition points, determined on the $16^{4}$ lattice, are listed in Table I. In Fig. 2 we draw the corresponding phase-transition line in the $\beta-\kappa$ plane.

It is already clear from Fig. 1 that the Higgs PT at

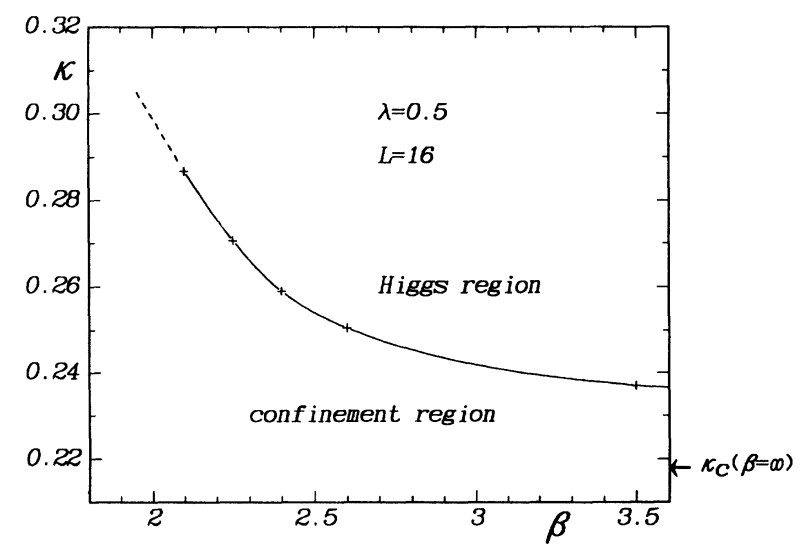

FIG. 2. The phase diagram of the SU(2) Higgs model at $\lambda=0.5$ on $16^{4}$ lattices. The positions of the phase-transition points are listed in Table $\mathrm{I}$. We indicate the transition point at $\beta=\infty$ [scalar $\mathrm{O}(4)$ theory]. The solid line ends at the most probable position of the critical point and the dashed line reflects the possible continuation allowed by the errors. 
TABLE I. Positions of the Higgs phase-transition points determined on the $16^{4}$ lattice. At $\beta=1.95$ the position of the specific-heat peak is indicated by an asterisk.

\begin{tabular}{ll}
\hline$\beta$ & \multicolumn{1}{c}{$\kappa_{\mathrm{PT}}$} \\
\hline 1.95 & $0.305(1)^{*}$ \\
2.1 & $0.28675(10)$ \\
2.25 & $0.27065(5)$ \\
2.4 & $0.25900(20)$ \\
2.6 & $0.25060(30)$ \\
3.5 & $0.23650(50)$ \\
\hline \hline
\end{tabular}

$\lambda=0.5$ is not a strong first-order phase transition anywhere. At some of the considered $\beta$ values we nevertheless detected metastability phenomena with small gaps $\Delta E$ depending on the lattice size. Here we present an analysis of these gaps for various $\beta$ and lattice sizes. The results are collected in Table II.

For $\beta=2.25$ we display in Fig. 3 a sequence of distribution functions $N(E)$ obtained on lattices $L=6,8,12$, and 16. The $\kappa$ values are always chosen such that the double-peak structure in $N(E)$ is approximately symmetric. On larger lattices a clear double-peak structure due to metastable states is seen, becoming more pronounced as the lattice size increases. For the $6^{4}$ lattice the distribution $N(E)$ is dome shaped without pronounced peaks. Here we tried the smoothing technique which was described in Sec. II C. The utility of this approach is demonstrated in Fig. 4 where we display the distribution $N^{*}\left(E^{*}\right)$ of the smoothed values $E_{j}^{*}$ for smoothing length $N_{S}=50$. Double peaks become clearly visible. We determined $E_{+}$and $E_{-}$from the positions of the maxima of the cubic spline interpolation curves, also
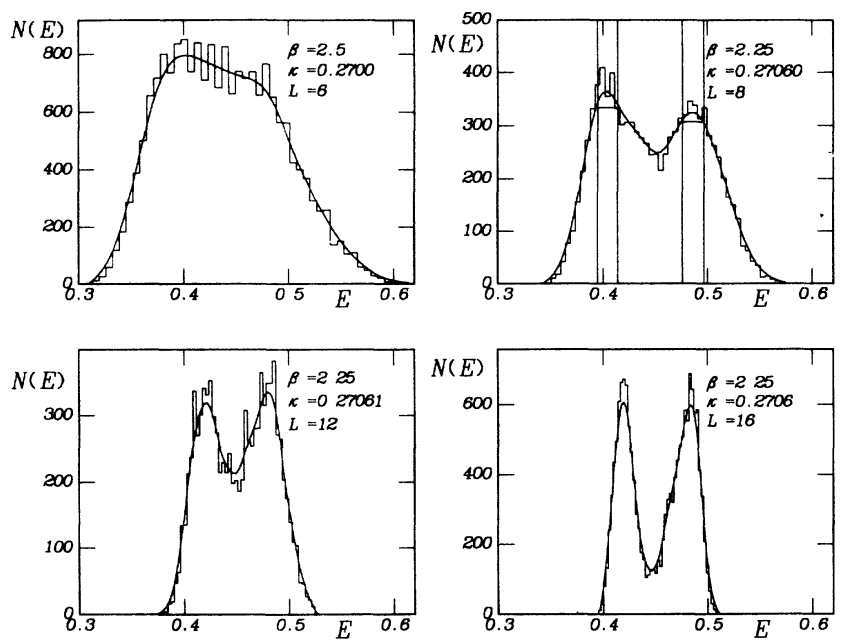

FIG. 3. A sequence of distribution functions $N(E)$ at $\beta=2.25$ on lattices of size $L=6,8,12$, and 16 . The curves are cubic spline interpolations. The vertical solid lines plotted for the $8^{4}$ lattice illustrate the error estimates of $E_{+}$and $E_{-}$.

shown in Figs. 3 and 4. The errors were estimated as follows. For runs with very high statistics the data were divided into several computer time bins and $E_{+}$and $E_{-}$ were determined for each bin from the position of the maxima of the spline interpolation functions to $N(E)$ or $N^{*}\left(E^{*}\right)$. The errors were calculated from the scattering of the positions of the maxima. We found that the error corresponds approximately to the width of the peaks as 95\% of the maximal height. This error estimate was adopted also for runs with a smaller statistics, when a bin

TABLE II. Positions $E_{+}$and $E_{-}$of metastable states and $\Delta E$ depending on $\beta, \kappa$, and $L$. Values of the gap marked with an asterisk have been obtained with the smoothing technique.

\begin{tabular}{cccccc}
\hline \hline$\beta$ & $L$ & $\kappa$ & $E_{-}$ & $E_{+}$ & $\Delta E_{+}-E_{-}$ \\
\hline 2.1 & 4 & 0.28350 & $0.420 \pm 0.015$ & $0.580 \pm 0.018$ & $0.160 \pm 0.033^{*}$ \\
& & 0.28400 & $0.421 \pm 0.014$ & $0.593 \pm 0.016$ & $0.172 \pm 0.030^{*}$ \\
& 6 & 0.28650 & $0.461 \pm 0.011$ & $0.604 \pm 0.021$ & $0.143 \pm 0.032^{*}$ \\
& & 0.28700 & $0.480 \pm 0.012$ & $0.620 \pm 0.012$ & $0.140 \pm 0.024^{*}$ \\
& 8 & 0.28650 & $0.476 \pm 0.008$ & $0.585 \pm 0.012$ & $0.109 \pm 0.020^{*}$ \\
& 12 & 0.28680 & $0.505 \pm 0.009$ & $0.608 \pm 0.010$ & $0.103 \pm 0.019^{*}$ \\
& & 0.28670 & $0.509 \pm 0.009$ & $0.590 \pm 0.007$ & $0.081 \pm 0.016$ \\
2.25 & 0.28680 & $0.501 \pm 0.008$ & $0.579 \pm 0.008$ & $0.078 \pm 0.016$ \\
& 16 & 0.28677 & $0.535 \pm 0.010$ & $0.595 \pm 0.005$ & $0.055 \pm 0.015$ \\
& 4 & 0.28680 & $0.535 \pm 0.010$ & $0.479 \pm 0.008$ & $0.060 \pm 0.015$ \\
& 6 & 0.27000 & $0.377 \pm 0.010$ & $0.487 \pm 0.011$ & $0.102 \pm 0.018^{*}$ \\
& 8 & 0.27000 & $0.384 \pm 0.006$ & $0.496 \pm 0.007$ & $0.082 \pm 0.014^{*}$ \\
& & 0.27060 & $0.405 \pm 0.010$ & $0.476 \pm 0.006$ & $0.090 \pm 0.011$ \\
& 12 & 0.27080 & $0.418 \pm 0.004$ & $0.478 \pm 0.006$ & $0.058 \pm 0.011$ \\
& & 0.27055 & $0.421 \pm 0.006$ & $0.483 \pm 0.004$ & $0.057 \pm 0.012$ \\
\hline \hline
\end{tabular}




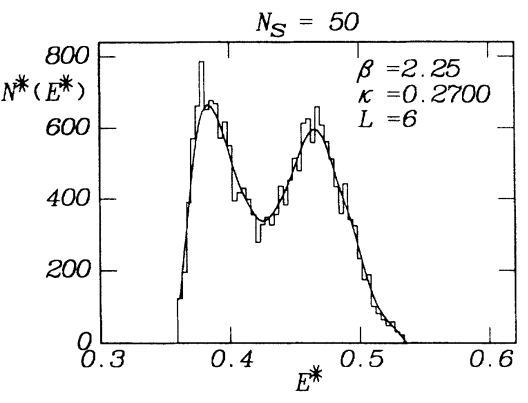

FIG. 4. Distribution $N^{*}\left(E^{*}\right)$ of the smoothed values $E^{*}$. The smoothing length is 50 sweeps.

analysis was not possible (see Fig. 3 for an example of this procedure). We remark that for different $\kappa$ the values of $E_{+}$and $E_{-}$are consistent within the errors, as long as both peaks are seen (see Table II), they are also nearly independent of a change of the smoothing length $N_{S}$. For some of our distribution functions we also used double Gaussians and found values of $E_{+}$and $E_{-}$consistent with the determination described above.

For $\beta=2.1$ and $L \leq 12$ the situation is analogous to $\beta=2.25$ and using distribution functions and the smoothing technique we again found metastable states on lattices of size $L=4,6,8$, and 12 and determined their positions. On the $16^{4}$ lattice we did not find clear double peaks in histograms even when utilizing the smoothing technique. The metastable states are only indicated by two shoulders in $N(E)$, whose positions (the values of $E_{+}$and $E_{-}$) can be determined quite reliably, however. Inspecting the evolution of $E_{i}$ with computer time we found long and smooth fluctuations; critical slowing down behavior of the system was apparent.

The situation for $\beta=2.4$ is comparable to that at $\beta=2.25$. We considered only $8^{4}$ and $16^{4}$ lattices and observed clear metastable behavior of the system in both cases. The gaps on both lattices are, within the errors, of the same size. We note that at $\beta=2.4$ the lifetime of the metastable states is even larger than at $\beta=2.25$ for the same lattice sizes, in spite of a smaller gap at $\beta=2.4$.

At $\beta=1.95,2.6$, and 3.5 we did not find signals for metastability even when using the smoothing technique on the $12^{4}$ and $16^{4}$ lattices. The distribution functions sampled appear to be close to Gaussian. Of course, this does not exclude the possible existence of slightly separated metastable states on even larger lattices.

In Fig. 5(a) we summarize our results for the gap $\Delta E$ at $\beta=2.1,2.25$, and 2.4 on lattices of various sizes. At $\beta=2.1$ we find a rapid decrease of the gap size with increasing lattice size $L$. The data are not good enough to perform a quantitative extrapolation of the gap to $L=\infty$, but the impression is that the gap probably vanishes in the thermodynamic limit. At $\beta=2.25$ we find a slowly decreasing gap size as we increase $L$. The gap stabilizes, however, for the largest lattice sizes considered. Here we conclude that the gap $\Delta E$ will probably survive in the thermodynamic limit. One can conclude this also from the distinct double-peak form of the distribution functions on the $12^{4}$ and $16^{4}$ lattices, Fig. 3. For $\beta=2.4$ the
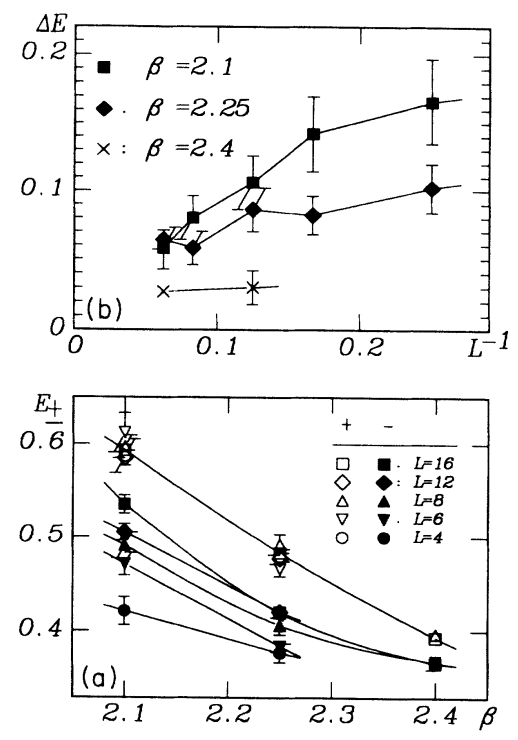

FIG. 5. (a) The gap $\Delta E$ as a function of $L^{-1}$ for several $\beta$. (b) Metastable states $E_{+}$and $E_{-}$. The lines are cubic spline interpolations of $E_{-}$for all lattice sizes, and of $E_{+}$(in order to maintain clarity) only for the $16^{4}$ lattice.

gap size seems to be rather independent of the lattice size and we conclude again that the phase transition is probably of first order. In Fig. 5(b) we plot the internal energies $E_{+}$and $E_{-}$of the metastable states as functions of $\beta$ for various lattice sizes $L$. First we note that the location of the upper state $E_{+}$, which is associated with the Higgs region, is rather independent of the lattice size. The finite-size dependence of the gap size is mainly caused by the finite-size dependence of the lower state $E_{\text {- }}$ associated with the confinement region. The curvature of $E_{-}$on the $16^{4}$ lattice as a function of $\beta$ is also remarkable, whereas the $E_{+}$values lie on a straight line. We finally note that on the $16^{4}$ lattice the maximal gap size is at $\beta \approx 2.25$. An interpolation of Fig. 5(b) of $E_{+}$and $E_{-}$on the $16^{4}$ lattice using cubic splines indicates that the gap for the $16^{4}$ lattice vanishes close to $\beta \approx 2.0$. The figure also suggests that the $\beta$ value at which the gap vanishes increases with increasing lattice size.

What can we say about the position of the critical point? With due caution with respect to the uncertainties associated with an interpolation to the thermodynamic limit, we conclude from the absence of metastable states at $\beta=1.95$ and from the indicated vanishing of the gap on the $16^{4}$ lattice around $\beta \approx 2.0$ (or higher), that the critical point lies above $\beta=1.95$. On the other hand, at $\beta=2.25$ the gap seems to persist for large $L$; therefore, we expect the end point to lie below this $\beta$ value. As seen in Table $I$ the crossover at $\beta=1.95$ and the Higgs PT points at $\beta=2.1$ and 2.25 on the $16^{4}$ lattice lie on an approximately straight line. Thus we expect that also the critical point lies on this line, i.e., between the points $(\beta, \kappa)=(1.95,0.305(1))$ and $(2.25,0.271(1))$. (The difference in $\kappa_{\mathrm{PT}}$ at $\beta=2.25$ and the increase of the error with respect to the values given in Table I reflects a possi- 
ble shift of $\kappa_{\mathrm{PT}}$ in the thermodynamic limit.) This localization of the critical point is considerably more precise than in earlier estimates, ${ }^{4}$ which suggests its position to be at some $\beta<1.5$. Similar change of the position of the critical point should be expected also for other $\lambda$, in particular for $\lambda=\infty$.

The other question is-Is there a tricritical point at some finite value of $\beta$ ? Our data in Fig. 5(b) show a rapid decrease of the gap size with $\beta$ between $\beta=2.25$ and $\beta=2.4$, and one may be tempted to estimate the location of a possible tricritical point at values of $\beta$ between 2.5 and 2.7. This reasoning however assumes the existence of a tricritical point at some finite $\beta=\beta_{\mathrm{TCP}}$ and a decrease of the gap size as some power of $\beta_{\mathrm{TCP}}-\beta$. This might not be true and a nonvanishing but exponentially small gap $\left(\sim e^{-\beta}\right)$ might persist up to the largest values of $\beta$ (as indicated by perturbation theory ${ }^{5}$ ). As we cannot rule out this possibility, our claim is that if there is a tricritical point at some finite coupling, then its most probable position is in the region $\beta=2.5-2.7$. One could perform a search using, e.g., the Monte Carlo renormalizationgroup method. We note that for the SU(2) Higgs model with the scalar fields in the adjoint representation this method indicates the presence of a tricritical point ${ }^{16}$ at similar $\beta$ values.

For completeness we present our results for the finitesize behavior of the specific heat $C$ and the modified fourth cumulant $V_{L}$ at values of $\beta=2.1$ and $\beta=2.25$ (at those values of $\beta$ we have the most complete data sets on various lattices). In Fig. 6(a) we present our data for the specific heat $C$, Eq. (5), at $\beta=2.1$. The peak of the specific heat on the $16^{4}$ lattice turns out to be so narrow
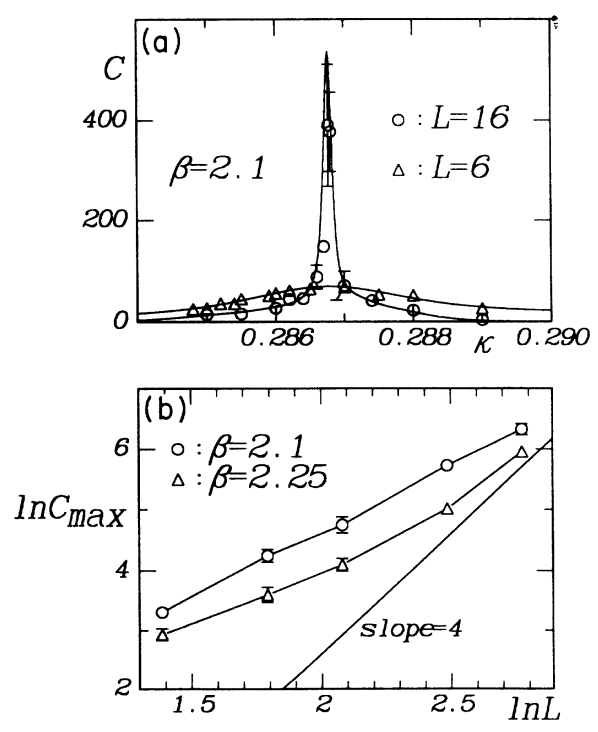

FIG. 6. (a) The specific heat at $\beta=2.1$ on $6^{4}$ and $16^{4}$ lattices. The symbols correspond to single MC runs and determinations of $C$ via Eq. (5). The curves have been obtained with the Ferrenberg-Swendsen method. (b) Dependence of the maximum values $C_{\max }$ of the specific heat on the lattice size $(\beta=2.1$ and $\beta=2.25$ ). The line corresponds to the value $\alpha / v=4$. that its maximum is not hit by the Monte Carlo (MC) runs. However, the curves corresponding to the determination of the specific heat with the FerrenbergSwendsen method, Eq. (10), predict a value of the maximum of the specific heat $C_{\max }$. For further illustration we display in Fig. 6(b) the values of $C_{\max }$ as obtained by the FS method, including an error estimate, as a function of the lattice size $L$ in logarithmic scales. The divergence of $C_{\max }$ with increasing volume is nicely observed for both values of $\beta$. At $\beta=2.25, C_{\max }$ increases for large $L$ according to (6) with $\alpha / v$ close to 4 , consistent with the earlier claim, that at $\beta=2.25$ the Higgs PT is of first order. The linear increase of $\ln C_{\max }$ with $\ln L$ at $\beta=2.1$ with a slope different from 4 can be considered as further indication that the phase transition at $\beta=2.1$ is very close to the critical point.

In Figs. 7(a) and 7(b) we compare the cumulant $V_{L}$ (determined by the FS method) at $\beta$ values 2.1 and 2.25 on various lattices. While $V_{L}$ increases with increasing lattice size we expect it to approach the value $\frac{2}{3}$ in the infinite-volume limit for $\kappa$ away from the phase-transition point, independent of $\beta$. However, for a first-order phase transition, we expect that its minimum value $V_{L}^{\text {min }}$ will stay smaller than $\frac{2}{3}$ in the infinite-volume limit on the infinite-volume phase-transition point. Our data at $\beta=2.25$ in Fig. 7(b) indeed show a slower increase of the minimum $V_{L}^{\text {min }}$ with increasing lattice than at $\beta=2.1$ in Fig. 7(a) (see, e.g., the change of $V_{L}^{\mathrm{min}}$ between $12^{4}$ and $16^{4}$ lattices); suggesting a value of $V_{\infty}^{\min }$ smaller than $\frac{2}{3}$ at $\beta=2.25$. The effect is however small, and the properties of $V_{L}$ alone do not allow a determination of the order of the phase transitions at the considered $\beta$ values.
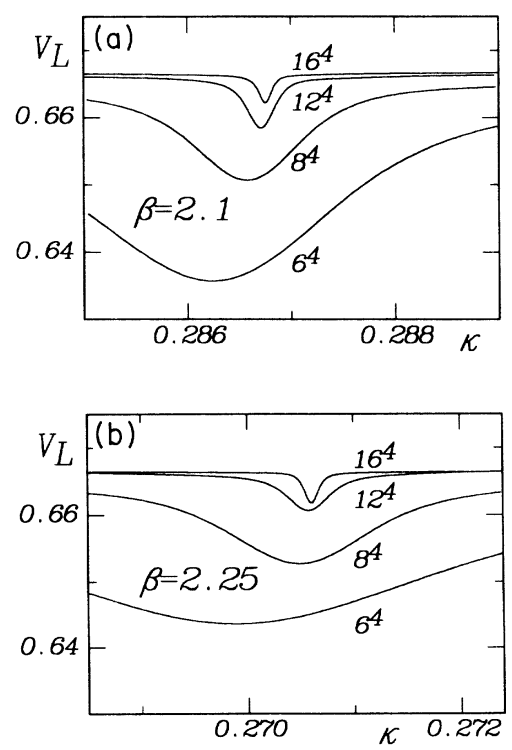

FIG. 7. The fourth-order cumulant $V_{L}$ at $\beta=2.1$ (a) and at $\beta=2.25$ (b) as obtained with the FS method. 


\section{CONCLUSIONS}

We performed a careful study of the properties of the Higgs PT at $\lambda=0.5$ at various values of $\beta$ by varying $\kappa$ in very fine steps. We conclude from the properties of metastable states observed in the distribution functions $N(E)$, that for $\beta=2.25$ and $\beta=2.4$ the Higgs PT is of first order. Here the gap size is practically unaffected by increasing the lattice size whereas for $\beta=2.1$ a strong decrease of the gap size is observable. We found that the critical end point lies in the interval $1.95<\beta<2.25$ and is probably very close to $\beta=2$. 1, i.e., at a value of $\beta$ substantially larger than suggested by earlier estimates. We studied also the specific heat and the modified fourthorder cumulant $V_{L}$ at $\beta=2.1$ and $\beta=2.25$ and found a consistent behavior. In the course of our investigation we applied the recently developed multihistogram method of Ferrenberg and Swendsen, which considerably improved our knowledge about the maximum of the specific heat and the minimum of the cumulant in the critical region. We were not able to clarify the existence of a possible tri- critical point, but assuming that it exists, it seems most likely to be in the region of $\beta$ values $2.5-2.7$. The Monte Carlo renormalization-group method would seem to offer more hope for resolving this question than do standard Monte Carlo simulations.

\section{ACKNOWLEDGMENTS}

We have benefited from discussions with $\mathbf{R}$. Baier, $\mathrm{K}$. Binder, V. Dohm, K. Jansen, H. A. Kastrup, C. B. Lang, and R. Shrock. A. M. Ferrenberg was indispensable with the implementation of the FS method. W.B., H.G.E., and T.N. thank the Center of Simulational Physics at the University of Georgia for its hospitality and support. We thank the Advanced Computational Methods Center of the University of Georgia and the computer center at the University of Bochum for providing the Cyber 205 computer time. Technical support by the computer centers of the Technische Hochschule Aachen and of the HLRZ Jülich is also gratefully acknowledged. This work was supported by the Deutsches Bundesministerium für Forschung und Technologie and by the Deutsche Forschungsgemeinschaft.
${ }^{1} \mathrm{~J}$. Jersák, in Lattice Gauge Theory-A Challenge in Large-Scale Computing, edited by B. Bunk, K. H. Mütter, and K. Schilling (Plenum, New York, 1986); R. E. Schrock, in Field Theory on the Lattice, proceedings of the International Symposium, Seillac, France, 1987, edited by A. Billoire et al. [Nucl. Phys. B (Proc. Suppl.) 4, 373 (1988)]; H. G. Evertz and M. Marcu, Report No. DESY 88-133, 1988 (unpublished).

${ }^{2}$ A. Hasenfratz and P. Hasenfratz, Phys. Rev. D 343160 (1986).

${ }^{3}$ R. Dashen and H. Neuberger, Phys. Rev. Lett. 50, 1897 (1983); P. Hasenfratz and J. Nager, Z. Phys. C 37, 477 (1988); A. Hasenfratz and T. Neuhaus, Nucl. Phys. B297, 205 (1988); A. Hasenfratz, K. Jansen, C. B. Lang, T. Neuhaus, and H. Yoneyama, Phys. Lett. B 199, 531 (1987); J. Kuti, in Proceedings of the XXIV International Conference on High Energy Physics, Munich, West Germany, 1988, edited by R. Kotthaus and J. Kühn (Springer, Berlin, 1989), and references therein; A. Hasenfratz, K. Jansen, J. Jersák, C. B. Lang, T. Neuhaus, and H. Yoneyama, Nucl. Phys. B317, 81 (1989).

${ }^{4} J$. Jersák, C. B. Lang, T. Neuhaus, and G. Vones, Phys. Rev. D 32, 2761 (1985).

${ }^{5}$ S. Coleman and E. Weinberg, Phys. Rev. D 7, 1888 (1973).

${ }^{6}$ H. Kleinert, Phys. Rev. Lett. 56, 69 (1986); F. Nill, thesis, Universität München, 1987 (unpublished); Freie Universität Berlin, reports, 1988 (unpublished).

${ }^{7}$ S. Aoki, I-H. Lee, and R. E. Shrock, Phys. Lett. B 207, 471 (1988).
${ }^{8}$ D. P. Landau and R. H. Swendsen, Phys. Rev. Lett. 46, 1437 (1981); P. Peczak and D. P. Landau, Phys. Rev. B 39, 11932 (1989).

${ }^{9}$ H. G. Evertz, J. Jersák, T. Neuhaus, and P. Zerwas, Nucl. Phys. B251 [FS13], 279 (1985).

${ }^{10} \mathrm{~K}$. Osterwalder and E. Seiler, Ann. Phys. (N.Y.) 110, 440 (1978); E. Fradkin and S. Shenker, Phys. Rev. D 19, 3682 (1979).

${ }^{11}$ G. Kalle and S. Wansleben, Comp. Phys. Commun. 33, 343 (1984); K. Jansen, J. Jersák, and T. Neuhaus, in Proceedings of the 1984 Conference on Cyber 205, Bochum, Germany, edited by $\mathrm{H}$. Ehlich et al. (Bochum University Press, Bochum, 1985), p. 151.

${ }^{12}$ For a review of the finite-size scaling, see $M$. N. Barber, in Phase Transitions and Critical Phenomena, edited by $\mathrm{C}$. Domb and J. L. Lebowitz (Academic, New York, 1983), Vol. 8 , p. 145.

${ }^{13}$ K. Binder and D. P. Landau, Phys. Rev. B 30, 1477 (1984).

${ }^{14}$ M. S. S. Challa, D. P. Landau, and K. Binder, Phys. Rev. B 34, 1841 (1986).

${ }^{15}$ M. Falcioni, E. Marinari, M. L. Paciello, G. Parisi, and B. Taglienti, Phys. Lett. 108B, 331 (1982); E. Marinari, Nucl. Phys. B235 [FS11], 123 (1984); A. M. Ferrenberg and R. H. Swendsen, Phys. Rev. Lett. 61, 2635 (1988); 63, 1195 (1989).

${ }^{16}$ R. Baier, C. B. Lang, and H.-J. Reusch, Nucl. Phys. B305 [FS23], 396 (1988). 